\title{
Congenital intestinal pseudo-obstruction associated with a giant platelet disorder
}

\author{
I Pollock, S J K Holmes, M A Patton, P A Hamilton, T E Stacey
}

\begin{abstract}
In this report we present a patient with the unique combination of chronic intestinal pseudo-obstruction and congenital thrombocytopenia with a nonspecific type of giant platelet disorder.
\end{abstract}

Congenital intestinal pseudo-obstruction is a rare disorder of gut motility, ${ }^{1}$ which may be 'idiopathic' or the result of a range of identified abnormalities of intestinal smooth muscle or its innervation. ${ }^{2}$ The commonest mode of inheritance appears to be autosomal recessive, ${ }^{3}$ although autosomal dominant, $\mathrm{X}$ linked dominant, and sporadic cases are described. ${ }^{34}$ There have been no previous reports of intestinal pseudo-obstruction occurring with a platelet disorder.

\section{Case report}

The male proband was the result of the first pregnancy of non-consanguineous, healthy 19 year old parents. There was no family history of gastrointestinal or haematological problems.

Dilated loops of bowel were noted on antenatal ultrasound in the third trimester, so a gastrointestinal problem was expected after birth. He was delivered at term by emergency caesarean section because of fetal distress. He was in good condition and no resuscitation was necessary. Birth weight was $4120 \mathrm{~g}$ (90th centile), length $52 \mathrm{~cm}$ (70th centile), and head circumference $36.5 \mathrm{~cm}$ (80th centile). No abdominal distension was observed at birth but within 24 hours abdominal $x$ rays showed multiple fluid levels and barium enema suggested a malrotation. At operation, malrotation with midgut volvulus was corrected and a narrowed segment of terminal ileum with proxi-

Department of Child Health, St George's Hospital, Cranmer Terrace, London SW17 ORE.

I Pollock, S J K Holmes, M A Patton, P A Hamilton, T E Stacey

Correspondence to Dr Pollock.

Received for publication 12 November 1990.

Accepted for publication 18 December 1990. mal dilatation was noted. Despite correction of the malrotation, symptoms of intestinal obstruction persisted, so on day 13 he underwent resection of the narrowed segment with a primary anastomosis. A rectal biopsy was taken at operation which showed an absence of ganglion cells, but Hirschprung's disease was thought unlikely owing to normal acetylcholinesterase staining. Detailed histology of the resected small bowel, in conjunction with abnormal intestinal motility studies, suggested a diagnosis of severe intestinal pseudo-obstruction of neural type. No evidence of an associated urinary tract abnormality was found.

Despite some improvement in tolerating enteral feeding, he continued to have biliary aspirates so a loop ileostomy was formed at 7 weeks. His clinical course was complicated by pyloric stenosis at 3 months for which he underwent a pyloromyotomy and a patent ductus arteriosus which was ligated at 6 months. Problems with the ileostomy led to a Bishop Koop colostomy being fashioned at 7 months. Repeated periods of partial enteral feeds resulted in severe pulmonary aspiration problems owing to gastric pooling. He has therefore been dependent on total parenteral nutrition which has been managed at home since the age of 2 years.

Thrombocytopenia was noted at birth with giant platelets seen on blood film. Coagulation studies and bone marrow aspirates were normal. A diagnosis of a non-specific giant platelet disorder was made by exclusion of known abnormalities of platelet function, such as the Bernard-Soulier syndrome, primary aggregation disorders, grey platelet syndrome, and storage pool diseases. Platelet counts have remained around $50 \times 10^{9} / 1$ with no clinical evidence of bleeding or bruising. Both parents have normal platelet numbers and function.

\section{Discussion}

This patient has a unique combination of congenital intestinal pseudo-obstruction with a giant platelet abnormality and thrombocytopenia. Owing to the negative family history this case may represent autosomal recessive inheritance, which has been 
reported for giant platelet disorders ${ }^{5}$ and many cases of intestinal pseudo-obstruction. ${ }^{3}$

Prenatal diagnosis has been offered to these parents by fetal blood sampling to detect platelet abnormalities, as the bowel abnormalities would be unrecognisable until late in pregnancy.

We would like to thank Dr M Powling for performing the platelet studies, Dr P Milla for the intestinal motility studies and advice, and Professor Lake for the histological studies.
1 Hyman PE, Tomomasa T, McDiarmid SV. Intestinal pseudoobstruction in childhood. In: Milla PJ, ed. Disorders of gastrointestinal motility in childhood. Chichester: John Wiley, 1988 . 73-80.

2 Lake BD. Observations on the pathology of pseudo-obstruction. In: Milla PJ, ed. Disorders of gastrointestinal motility in childhood. Chichester: John Wiley, 1988:81-90.

3 Vargas JH, Sachs P, Ament ME. Chronic intestinal pseudoobstruction syndrome in pediatrics. $\mathcal{F}$ Pediatr Gastroenterol Nutr 1988;7:323-32.

4 Wiswell T, Rawlings J, Wilson J, Pettet G. Megacystis-microcolon-intestinal hypoperistalsis syndrome. Pediatrics 1979;63: 805-8.

5 Hardisty RM. Platelet disorders. In: Hoffbrand AV, Lewis SM, eds. Postgraduate haematology. Oxford: Heinemann, 1989: 598-626. 